\title{
Rosenkrieg am Ende einer Zwangsehe - Die Entwicklungen im Sudan nach der Unabhängigkeit des Südsudan
}

\author{
Gerald Hainzl*
}

\begin{abstract}
South Sudan gained independence from Sudan in July 2011. While the people in both Sudan and South Sudan thought that a kind of peace dividend would lead to an improvement of their lives, these hopes have not been fulfilled. The bilateral relationship is all but good, and solutions for contentious issues like borders, citizenship and the status of Abyei are still pending. Despite separation and a "War of the Roses", Sudan and South Sudan are still bound by mutual economic and social ties. Furthermore, both countries have to cope with home-grown problems and fierce economic situations due to their oil export dependence and the resulting abilities to block each other's oil extraction or transportation.
\end{abstract}

Keywords: Sudan, South Sudan, independence, bilateral relations, conflict, domestic challenges Sudan, Südsudan, Unabhängigkeit, bilaterale Beziehungen, Konflikt, innerstaatliche Probleme

\section{Einleitung ${ }^{1}$}

$\mathrm{M}$ it der Unabhängigkeit der Republik Südsudan haben sowohl der Südsudan als auch der Sudan große Hoffnungen verknüpft, wenngleich aus meist unterschiedlichen Gründen. Beide Seiten hegten jedoch die Hoffnung, dass eine sogenannte Friedensdividende relativ rasch die Lebensumstände verbessern wird. ${ }^{2}$ Diese Hoffnung wird mehr und mehr enttäuscht. Weder werden die sehr hohen Erwartungen der Menschen im Südsudan erfüllt, noch erreichte der Sudan eine wesentliche Verbesserung seiner internationalen Beziehungen. Mehrere internationale Missionen werden noch länger als stabilisierende Elemente der Staatengemeinschaft vor Ort tätig bleiben müssen. Der 9. Juli 2011, der Tag der Unabhängigkeit des Südsudan, sollte deshalb nicht als Ende eines gewaltsamen Konflikts begriffen, sondern als Beginn eines längerfristigen Engagements zur Konfliktprävention verstanden werden.

\section{Historischer Überblick}

1956 wurden Sudan und Südsudan als gemeinsamer Staat in die Unabhängigkeit entlassen, obwohl die Kolonialpolitik des Vereinten Königreichs von Großbritannien und Nordirland zwischen 1883 und 1956 mehr oder weniger getrennte Administrationen geschaffen hatte. Als 1953 die innere Verwaltung übergeben wurde, wurden praktisch kaum Posten mit Südsudanesen besetzt. ${ }^{3}$ Bereits zwei Jahre später rächte sich dies, als Soldaten des Equatorial Corps, die durch Soldaten aus dem Nor-

* Dr. Gerald Hainzl forscht und lehrt am Institut für Friedenssicherung und Konfliktmanagement an der Landesverteidigungsakademie in Wien.

1 Der folgende Text beruht auf einem mehrjährigen Forschungsprojekt des Instituts für Friedenssicherung und Konfliktmanagement. Einige Ideen und analytische Ableitungen wurden bereits in Publikationen des Instituts, insbesondere in den Reihen IFK aktuell und IFK Monitor, dargelegt.

2 Hainzl, Gerald und Pospisil, Jan. Sudan, Südsudan. 2012. In: WertherPietsch, Ursula und Ritzer, Thomas. Failed States - Staatsaufbau als Konfliktprävention. Neuer Wissenschaftlicher Verlag: Wien - Graz.

3 Rout Biel, Melha. 2004. Modernisierung der Bildung und Verwaltung im Sudan. Ein Land zwischen Bürgerkriegen, Diktaturen und Fremdherrschaft. Lit Verlag: Münster. S. 85. den ersetzt werden sollten, meuterten und desertierten. ${ }^{4}$ Sie bildeten den Kern jener Rebellen, die von 1963 bis 1972 unter dem Namen Anya Nya (Schlangengift) gegen die Regierung in Khartoum sowie die Dominanz des Nordens kämpften.

Im Rahmen von Friedensverhandlungen wurde dem Süden zwar eine Autonomieregelung zugestanden, diese führte jedoch nicht zur angestrebten politischen und wirtschaftlichen Gleichberechtigung. Eine gewisse ökonomische Erholung war zwar auch im Süden erkennbar, in der Staatsverwaltung existierte jedoch keine gleichberechtigte Mitwirkung. Die Beziehungen zwischen Nord und Süd verschlechterten sich daher schon in den 1970er Jahren. 1983 eskalierte der Bürgerkrieg von Neuem. Ausschlaggebend waren dafür im Wesentlichen zwei Gründe: Wirtschaftlich waren die Entdeckung von Erdölvorkommen auf dem Gebiet des Südsudan 1978 sowie die Entscheidung der Regierung, eine Raffinerie im Norden des Landes zu bauen, Konfliktpunkte. Die Menschen im Süden befürchteten, vom neuen Wohlstand ausgeschlossen zu werden. Politisch schürten im Jahr 1983 die Aussetzung über Autonomie und Islamisierung des Rechtssystems im gesamten Staatsgebiet die Ängste der Menschen im Süden, womöglich zentrale Werte ihrer religiösen und kulturellen Identität zu verlieren. ${ }^{5}$

Erst im Januar 2005 konnte der Bürgerkrieg mit einem umfassenden Friedensabkommen (Comprehensive Peace Agreement $\mathrm{CPA})^{6}$ formal beendet werden. Bis zu diesem Zeitpunkt wurden zwei Millionen Menschen Opfer der Auseinandersetzung. Der Tod des charismatischen John Garang de Mabior im Juli 2005 stellte eine Wende in der südsudanesischen Politik dar. War Garang noch von einem „neuen Sudan“ überzeugt, änderte sich die Position unter seinem Nachfolger Salva Kiir Mayardit.

4 Pabst, Martin. 2004. Krieg und Frieden im Sudan. In: Feichtinger, Walter (Hrsg.): Afrika im Blickfeld. Kriege - Krisen - Perspektiven. Nomos Verlagsgesellschaft: Baden-Baden. S. 136f.

5 Für eine detaillierte Darstellung des Bürgerkriegs im Südsudan siehe Deng D. Akol Ruay. 1994: The Politics of the two Sudans. The South and the North 1821-1969. Nordiska Afrikainstitutet. Dezember, sowie Holt, P.M. und Daly, M.W. 2011. A History of the Sudan: From the Coming of Islam to the Present Day (6th Edition). Longman: London.

6 The Comprehensive Peace Agreement Between The Government of The Republic of The Sudan and The Sudan People's Liberation Movement/ Sudan People's Liberation Army, http://unmis.unmissions.org/Portals/UNMIS/ Documents/General/cpa-en.pdf. 
Offiziell wurde immer noch an der Einheit des Staates festgehalten, allerdings wurde die Zeit bis zum Referendum nicht dafür genutzt, um den Gedanken an einen gemeinsamen Staat im Bewusstsein der Südsudanesen zu verankern. Selbst der Norden entwickelte erst wenige Monate vor dem Referendum Aktivitäten, um die Einheit des Landes innerhalb der Bevölkerung zu bewerben. Es war daher wenig verwunderlich, dass sich die überwältigende Mehrheit der südsudanesischen Bevölkerung (98,83\%) bei der Abstimmung im Januar 2011 für die Unabhängigkeit des Südsudan aussprach.

\section{Die Zeit nach dem Referendum}

Auch die sechs Jahre zwischen dem Friedensabkommen 2005 und der Volksabstimmung 2011 blieben in friedenspolitischer Hinsicht ungenutzt. Überlegungen, wie man anstehende Probleme, die im Falle einer Trennung auftreten würden, bereits im Vorfeld lösen könnte, wurden nicht angestellt. So sah das CPA etwa vor, dass die südsudanesische Bevölkerung die Möglichkeit haben sollte, über eine Abspaltung abzustimmen, ermöglichte es aber aufgrund anderer Vereinbarungen nicht, dass im Südsudan aktiv Kampagnen für einen eigenen Staat gestartet werden konnten. Ein Grund dafür liegt vermutlich in Punkt 1.1 den Agreed Principles des CPA, in dem es heißt, „[t]hat the unity of the Sudan ... is and shall be the priority of the Parties and that it is possible to redress the grievances of the people of South Sudan and to meet their aspirations within such a framework." Gleiches gilt für Punkt 2.4.2, der folgende Bestimmung beinhaltet: „The parties shall work ... making the unity of Sudan attractive to the people of South Sudan." Dadurch konnten jene Fragen nicht offen angesprochen und diskutiert werden, die im Falle einer Trennung vermutlich zu Problemen zwischen den beiden Staaten führen würden. Im Besonderen betraf das die Frage der Staatsbürgerschaft und der Aufenthaltsrechte der jeweiligen Bürger, Grenzfragen, wie die Zugehörigkeit der Provinz Abyei sowie Fragen der Ölförderung und des Öltransits.

\section{Die Suche nach Lösungen für zwischenstaatliche Probleme}

\subsection{Aktuelle Konfliktfelder}

Ein wesentliches Konfliktfeld, Staatsbürgerschaft und Aufenthaltsrechte, konnte noch vor der Unabhängigkeit durch eine Fristverlängerung entschärft werden, um die Ausarbeitung einer nachhaltigen politischen Lösung zu erreichen. Dennoch bleibt die Frage der Rechte der Bürger im jeweils anderen Staat nach wie vor ungelöst. Insgesamt sollen sich noch ca. 500.000 Südsudanesen im Sudan und ca. 80.000 Sudanesen im Südsudan aufhalten. ${ }^{7}$

Insbesondere der Streit um Erdöl dominierte nach der Unabhängigkeit die Beziehungen zwischen den beiden Staaten im wirtschaftlichen Bereich, wobei die Frage der Grenzziehung und jene des Erdöltransits gleichermaßen eine Rolle spielten. Es ging/geht bei strittigen Grenzfragen zwar auch um Land,

7 South Sudanese refugees flown home from Khartoum, http://www.bbc. co.uk/news/world-africa-18059276, abgerufen am 08.01.2013.
Landrechte und die staatliche Zugehörigkeit verschiedener nomadisierender und sesshafter Gruppen. Die politischen und akademischen Diskurse wurden jedoch fast ausschließlich von den ökonomischen Fragen dominiert. Dies wurde besonders im Fall der Provinz Abyei deutlich.

In Abyei werden zwar nur etwa 0,6\% des gesamten Erdölertrags (Sudan und Südsudan) gefördert, trotzdem wurde die Provinz zum Kristallisationspunkt der Auseinandersetzungen. Noch vor der Unabhängigkeit, und zwar im Mai 2011, drangen sudanesische Streitkräfte in die Provinz ein. Nach Angaben verschiedener Hilfsorganisationen flüchteten daraufhin etwa 100.000 Menschen aus Abyei. Eine 4.200 Mann starke VN-Truppe wurde eingesetzt und konnte die Gefahr einer militärischen Auseinandersetzung vorerst bannen. Die UNISFA (United Nations Interim Security Force for Abyei) war nur für sechs Monate geplant und sollte im Wesentlichen den Abzug der sudanesischen Streitkräfte und der Sudanesischen Volksbefreiungsarmee (Sudan People's Liberation Army, SPLA) verifizieren. ${ }^{8}$ Im Dezember 2011 wurde das Mandat der Mission zum ersten Mal verlängert und um Aufgaben im Bereich der Normalisierung der Grenzbeziehungen zwischen Sudan und Südsudan ergänzt. Hierzu zählten z.B. Maßnahmen zum Aufbau bilateraler Mechanismen sowie allgemeiner vertrauensbildender Maßnahmen. ${ }^{9}$ Der VN-Sicherheitsrat verlängerte im November 2012 die Mission erneut bis zum 31. Mai 2013. ${ }^{10}$ Nach derzeitigem Stand könnte eine Interimsadministration zur Lösung des Konflikts um Abyei beitragen. Beide Konfliktparteien haben diesem Vorschlag, der vom African Union Peace and Security Council (AUPSC) lanciert wurde, bereits im Juli 2011 grundsätzlich zugestimmt, diesen aber immer noch nicht umgesetzt. Der Vorschlag sieht zudem vor, im Oktober 2013 ein Referendum über den endgültigen Status von Abyei abzuhalten. ${ }^{11}$

Auch der Angriff südsudanesischer Truppen auf die Ölfelder in der Nähe der Ortschaft Heglig (im Südsudan Panthou genannt) im April 2012 verdeutlichte einmal mehr das vorhandene Konfliktpotenzial zwischen den beiden Staaten. Interpretationen hinsichtlich der dem Angriff zugrunde liegenden Ratio gibt es mehrere. Diese reichen von einer Auslegung als Revanche für den sudanesischen Einmarsch in Abyei im Jahr zuvor über ökonomische Gründe (Sicherung der Heglig-Ölfelder) bis zu Gerüchten, dass der südsudanesische Präsident Salva Kiir Mayardit auf Grund von realen oder perzipierten Putschgefahren Stärke demonstrieren wollte. ${ }^{12}$

Monate vor dem Einmarsch in Heglig, im Januar 2012, hatte der Südsudan seine Ölexporte über die Pipeline durch den Sudan eingestellt. ${ }^{13}$ Dieser Maßnahme war ein Streit über die

8 Resolution 1990 (2011), Adopted by the Security Council at its 6567th meeting, on 27 June 2011.

9 Resolution 2024 (2011), Adopted by the Security Council at its 6683rd meeting, on 14 December 2011.

10 Resolution 2075 (2012), Adopted by the Security Council at its 6864th meeting, on 16 November 2012.

11 S. Sudan links formation of Abyei interim administration to final status, http://www.sudantribune.com/spip.php?article45054, abgerufen am 03.01.2013.

12 Unbestritten scheint zu sein, dass beide Präsidenten, Salva Kiir und Omar Hassan al-Bashir, von dieser Situation innenpolitisch profitieren konnten.

13 Statement by H.E. Salva Kiir Mayardit, President of the Republic of South Sudan to the National Legislature on the current oil crisis January 23 2012, http://www.sudantribune.com/Statement-by-H-E-Salva-Kiir,41381, abgerufen am 03.01.2013. 
Konfiszierung südsudanesischen Öls durch den Sudan vorausgegangen. Der Sudan erhob den Vorwurf, der Südsudan habe Transitgebühren nicht bezahlt. ${ }^{14}$ Bereits im Juli 2011 hatte Präsident Salva Kiir martialisch angekündigt, der Südsudan könne länger ohne die Einnahmen aus der Ölproduktion auskommen und auch noch während der nächsten Jahre darauf verzichten.: „But we fought for 21 years without oil and we can still go for 3 years until we build our own oil infrastructure." ${ }^{15}$ Doch blieb dies nicht mehr als eine Drohgebärde. Der Südsudan hatte nicht nur seine finanziellen Möglichkeiten überschätzt. ${ }^{16}$ Auch der Druck von außen, eine Lösung im Erdölstreit zu finden, insbesondere von den USA, aber auch von China, einem der wichtigsten Abnehmer des sudanesischen Erdöls, wurde zunehmend stärker. Der Streit konnte Anfang August 2012 zumindest entschärft werden, einen Monat später verständigten sich die beiden Präsidenten über die Wiederaufnahme der Exporte, die im Dezember 2012 nach einem Jahr Pause wieder starten sollten. ${ }^{17}$

Die Fragilität der Beziehungen sowie das fehlende gegenseitige Vertrauen der handelnden Personen auf beiden Seiten zeigen sich auch in der problematischen Art und Weise der Umsetzung der geschlossenen Abkommen. Im September 2012 einigten sich die beiden Präsidenten beispielsweise auf eine entmilitarisierte Pufferzone entlang der gemeinsamen Grenze, nicht zuletzt, um die wechselseitigen Anschuldigungen, dort würden Rebellengruppen unterstützt, mit einer für beide Seiten akzeptablen Lösung zu entschärfen. Khartoum beschuldigt den Südsudan, dass dieser die Sudanese People's Liberation Movement-North (SPLM-N) in Süd-Kordofan unterstützt und hat die Anschuldigungen bisher nicht zurückgenommen, obwohl Juba diese stets zurückweist. Die Implementierung der entmilitarisierten Zone scheiterte jedoch bislang auch am Erdölstreit.

Eine internationale Überwachung problematischer Grenzgebiete wäre eine Möglichkeit gewesen, bereits zum Zeitpunkt der Unabhängigkeit des Südsudan konfliktive Entwicklungen einzudämmen. Eine Informationsplattform für beide Seiten hätte bereits damals und könnte noch heute zur Vertrauensbildung beitragen und auch künftig mögliche militärische „Kurzschlusshandlungen“ verhindern. ${ }^{18}$ Allerdings werden diesbezügliche Ansätze von den Konfliktparteien teilweise abgelehnt und erschweren internationales Krisen- und Konfliktmanagement.

\subsection{Direktverhandlungen ohne nennenswerten Durchbruch}

Bei neuerlichen Direktverhandlungen zwischen Omar Hassan al-Bashir und Salva Kiir Mayardit Anfang Januar 2013 in

14 North confiscates South Sudan oil, http://www.upstreamonline.com/live/ article297965.ece, abgerufen am 03.01.2013.

15 South Sudan's Kiir talks tough on oil, Abyei, http://www.sudantribune.com/ South-Sudan-s-Kiir-talks-tough-on,39591, abgerufen am 03.01.2013.

16 Verschiedenen Schätzungen zufolge machen die Einnahmen aus dem Ölexport ca. $98 \%$ des südsudanesischen Budgets aus.

17 Oil to flow again: Pagan, http://www.radiomiraya.org/index.php/southsudan-news/10339-oil-to-flow-again-pagan\#gsc.tab=0, abgerufen am 03.01.2013.

18 Hainzl, Gerald und Feichtinger, Walter. Sudan und Südsudan 2012. IFK Monitor. Jänner 2012.
Addis Abeba konnten die beiden Präsidenten zwar in einigen Punkten eine Übereinstimmung erzielen, insgesamt sind sie aber nach wie vor weit davon entfernt, alle strittigen Fragen nachhaltig zu klären. Die AU ließ verlauten, dass zumindest hinsichtlich Abyei in den Punkten Abyei Area Administration, Abyei Area Council und Abyei Area Police Service eine Einigung erzielt werden konnte. Nach wie vor ist unklar, ob es im Oktober 2013 zu einem Referendum in Abyei kommen wird. Der Sudan stimmt einem Urnengang ohne die Teilnahme von Angehörigen der Misseriya ${ }^{19}$ nicht zu, obwohl gerade diese Variante vom African Union High-Level Implementation Panel (AUHIP) und vom African Union Peace and Security Council (AUPSC) unterstützt werden würde. ${ }^{20}$

Khartoum und Juba werden die Demarkierung jener Grenzabschnitte fortsetzen, die bereits außer Streit standen und ca. 80\% der gemeinsamen Grenze ausmachen. Jene Bereiche, die nach wie vor umstritten sind, sollen bei einem späteren Präsidententreffen diskutiert werden.

\subsection{Internationales Krisen- und Konfliktmanagement}

Als der Sicherheitsrat der VN im Jahr 2004 mit der Resolution 1547 (2004) ${ }^{21}$ die United Nations Advance Mission in the Sudan (UNAMIS) beschloss, war dies eine Maßnahme zur Unterstützung des politischen Prozesses im Sudan. Die Mission sollte ein Friedensabkommen zwischen Nord und Süd ermöglichen. Auf diese Weise signalisierte die VN auch ihre Bereitschaft, sich im Sudan über einen gewissen Zeitraum zu engagieren. Die gewaltsame Eskalation des Konflikts in Darfur führte dazu, dass der Sicherheitsrat bereits nach wenigen Wochen mit der Resolution 1556 (2004) ${ }^{22}$ das Aufgabenspektrum der Mission erweiterte. ${ }^{23}$ Nach der Unterzeichnung des CPA im Januar 2005 wurde mithilfe der Resolution 1590 (2005) ${ }^{24}$ UNAMIS in die Kapitel-VII-Mission UNMIS (United Nations Mission in the Sudan) überführt. Ihre Aufgaben umfassten:

- die Implementierung des CPA zu unterstützen,

- bei der freiwilligen Rückkehr von Flüchtlingen und intern Vertriebenen zu helfen und diese zu koordinieren,

- den Parteien des CPA bei der Entminung sowie allgemeine technische Unterstützung und Koordinierungshilfe zu leisten.

19 Die Misseriya sind Nomaden, deren Wanderbewegungen grenzüberschreitend sind. Daher wird darüber gestritten, ob sie tatsächlich wahlberechtigt sind, da sie je nach Interpretation, entweder in Abyei ansässig sind, oder eben nicht.

20 Bashir-Kiir summit ends with agreement on implementation timeframe but no breakthrough, http://sudantribune.com/spip.php?article45089, abgerufen am 07.01.2013.

21 Resolution 1547 (2004). Adopted by the Security Council at its 4988th meeting, on 11 June 2004, http://www.mpil.de/shared/data/pdf/ resolution_1547.pdf.

22 Resolution 1556 (2004). Adopted by the Security Council at its 5015th meeting, on 30 July 2004.

23 Die Eskalation in Darfur steht in direktem Zusammenhang mit den Fortschritten in den Friedensverhandlungen zwischen Nord- und Südsudan. Im Norden existierte die Angst, nicht nur den Süden, sondern auch den Westen und damit eine weitere rohstoffreiche Region des Landes zu verlieren. Bis heute fürchten die Eliten in Khartoum die sogenannte „Vierteilung“, denn auch im Ostsudan hat es in der Vergangenheit antisudanesische Tendenzen gegeben.

24 Resolution 1590 (2005). Adopted by the Security Council at its 5151st meeting, on 24 March 2005. 
Darüber hinaus sollte UNMIS einen Beitrag zu den internationalen Anstrengungen zum Schutz und zur Förderung der Menschenrechte leisten, wobei Flüchtlingen, Binnenvertriebenen, Frauen und Kindern besondere Aufmerksamkeit geschenkt werden sollte.

Das Mandat von UNMIS endete am 9. Juli 2011 mit der Unabhängigkeit des Südsudan. Bereits zu diesem Zeitpunkt wäre eine Mission entlang der gemeinsamen Grenze möglich gewesen. Sie hätte vermutlich zur Stabilisierung der Grenzregion beitragen können. Der Sudan (Khartoum) lehnte jedoch sowohl eine Verlängerung von UNMIS als auch ein neues Mandat ab. Daraufhin beschloss der Sicherheitsrat statt einer Mission, die beide Länder umfasste, mit der Resolution 1996 (2011) die United Nations Mission in the Republic of South Sudan (UNMISS). Ziel dieser Mission ist unter anderem die Konsolidierung des Friedens und der langfristige Staatsaufbau im Südsudan. Der Südsudan soll zudem in den Bereichen Konfliktprävention, Konfliktentschärfung sowie Konfliktlösung und Schutz der Zivilbevölkerung Hilfe erhalten. Die Unterstützung der Regierung beim Kapazitätsaufbau zur Schaffung von Sicherheit und Rechtsstaatlichkeit sowie die Stärkung des Sicherheits- und Justizapparates im Rahmen der nationalen Selbstbestimmung gehören ebenfalls zu ihrem Aufgabenspektrum. Einen Beitrag in diesem Bereich liefert auch die Europäische Union (EU). Im Rahmen der European Union Aviation Security Mission (EUAVSEC South Sudan) soll die Sicherheit am Juba International Airport verbessert und gestärkt werden. ${ }^{25}$

UNISFA (siehe oben) bleibt die einzige Mission, die sowohl den Sudan als auch den Südsudan umfasst. UNMISS ist nur im Südsudan disloziert. Im Sudan, in Darfur, ist nach wie vor die African Union/United Nations Hybrid Operation in Darfur (UNAMID) aktiv. Kämpfe, die 2003 in Darfur zwischen Rebellengruppen und regierungstreuen Milizen ausgebrochen waren, führten zu einem militärischen Engagement der Afrikanischen Union (AU), nachdem von dieser ein Waffenstillstand (Humanitarian Ceasefire Agreement) vermittelt worden war. Aufgrund der fortgesetzten Gewalt gegen die Zivilbevölkerung beschloss die AU im Oktober 2004, die African Union Mission in Sudan (AMIS) personell aufzustocken. Möglich wurde dies durch die finanzielle Unterstützung der EU. Im Juli 2007 wurde schließlich vom VN-Sicherheitsrat mit der Resolution 1769 (2007)26 ein Mandat für knapp 20.000 Soldaten und ca. 3.800 zivile Kräfte erteilt. UNAMID, die erste Hybridmission von AU und VN, konnte dazu beitragen, dass das Gewaltniveau in der Region trotz aller Unzulänglichkeiten stark sank und sich gewaltsame Auseinandersetzungen zwischen Sudan und Südsudan nicht nach Darfur verlagern konnten, sei es direkt oder in Form von Stellvertreterkonflikten.

25 Siehe dazu EUAVSEC South Sudan, http://www.consilium.europa.eu/ eeas/security-defence/eu-operations/euavsec-south-sudan, abgerufen am 04.01.2013.

26 Resolution 1769 (2007). Adopted by the Security Council at its 5727th meeting, on 31 July 2007

\section{Innerstaatliche Herausforderungen}

Abseits der bilateralen Probleme sind beide Staaten mit inneren Herausforderungen konfrontiert. Bereits lange vor der Unabhängigkeit wurde für den Südsudan ein Ausbruch interethnischer Konflikte prognostiziert. ${ }^{27}$ Allerdings werden die Ursachen für diese Konflikte weniger in ethnischen als in ökonomischen Problemen gesehen. Die südsudanesische Gesellschaft hat große Erwartungen in die Eigenstaatlichkeit gesetzt, die von der Regierung bislang nicht erfüllt werden können. Nach dem Abebben der Unabhängigkeitseuphorie wird sich vermehrt eine tiefe Spaltung bemerkbar machen, die entlang ethnischer Identitäten verlaufen kann, aber nicht zwingend muss. Der frühere gemeinsame Feind im Norden kann nicht mehr länger als Sündenbock für Versäumnisse verantwortlich gemacht werden. Die südsudanesische Regierung wird daher Projekte, wie etwa im Bereich Infrastruktur, mit hoher Sichtbarkeit und großem Nutzen für weite Teile der Bevölkerung brauchen, um die Erwartungshaltung hinsichtlich einer Friedensdividende zu befriedigen.

Nach dem Abschluss des CPA 2005 hat auch der Aufbau eines Staatswesens begonnen. Einige Fortschritte sind zu erkennen, doch ist der Südsudan noch weit von einem politischen „Anstaltsbetrieb“28 entfernt. Die Verwaltungsstrukturen funktionieren nur bedingt und sind noch nicht in der Lage, Autorität über das gesamte Staatsgebiet auszuüben. Die Loyalitäten der Soldaten innerhalb der SPLA beschränken sich nach wie vor auf ihre Kommandanten. Daher sind einerseits Sicherheitsfragen und andererseits strukturelle Maßnahmen zum Aufbau von verlässlichen Sicherheitsstrukturen in den kommenden Jahren unerlässlich. Aber auch eine Verkleinerung der SPLA auf ein Maß, das der Größe und Bevölkerungsanzahl des Staates entspricht, sowie Maßnahmen zur Entwicklung wirtschaftlicher Perspektiven für demobilisierte Soldaten sollten möglichst rasch in Angriff genommen werden. Die fehlende staatliche Autorität zeigt sich auch in der hohen Anzahl an Viehdiebstählen. Da der Brautpreis im Südsudan vorwiegend in Rindern bezahlt wird und sowohl der Rinder- als auch der Brautpreis in den letzen Jahren stark angestiegen sind, hat sich Viehdiebstahl zu einem profitablen Wirtschaftszweig entwickelt. Der Organisationsgrad der Viehdiebe lässt den Schluss zu, dass nicht die Tradition, sondern Bereicherung im Vordergrund steht. Es gibt Gerüchte, dass auch Lokalpolitiker involviert sind. Die große Zahl an unzufriedenen jungen Männern, die aufgrund der hohen Kosten nicht heiraten können, könnte künftig destabilisierend wirken. ${ }^{29}$

Der ökonomische Schaden, der durch das Aussetzen der Erdöllieferungen durch den Südsudan entstanden ist, ist in beiden Staaten enorm. Die fehlenden Einnahmen können in keiner

27 Die meisten Studien dazu sind bloße Einschätzungen und beruhen nicht auf empirischem Datenmaterial. Siehe dazu Schilling, Sonja. 2012. „Ethnische Konflikte“ im Südsudan? Eine Auseinandersetzung mit sozialwissenschaftlichen Darstellungen von Konflikten im Südsudan und deren Sinnhaftigkeit. Diplomarbeit Universität Wien.

28 Vgl. Weber, Max. 1972: Wirtschaft und Gesellschaft. Kapitel 1. Soziologische Grundbegriffe. § 17. Mohr Siebeck: Tübingen. 5. Auflage. S. 29.

29 Siehe z.B. South Sudan cow theft leaves more than 200 dead, http://english.al-akhbar.com/node/5097, abgerufen am 09.01.2013, oder South Sudan suffers as cattle rustling intensifies, http://www.africanfarming.net/comnovapc-menu-uploader/livestock/cattle/south-sudan-suffers-as-cattle-rustling-continues, abgerufen am 09.01.2013. 
Weise kompensiert werden. Beide Staaten sind finanziell stark unter Druck geraten und waren bereits in der ersten Hälfte 2012 auf Kredite von externen Geldgebern angewiesen. ${ }^{30}$

Der Sudan ist aber nicht nur wirtschaftlich unter Druck. Viele innenpolitische Probleme, die nichts mit der Unabhängigkeit zu tun haben, wirken destabilisierend auf das Land. Die regierende Partei, National Congress Partei (NCP), muss zwar nicht um ihre Macht fürchten, da sie von den diversen Oppositionsgruppen kaum herausgefordert werden kann. Allerdings könnte die NCP durch innere Auseinandersetzungen geschwächt werden. In der Zeit nach dem Verlust des Südens blieb die Partei aber relativ homogen. ${ }^{31}$ Die drohende Eskalation des Konflikts mit dem Südsudan hat Präsident al-Bashir sicherlich genützt, da er einen Schulterschluss in den Reihen der politischen Elite zur Folge hatte. Allerdings steht zu erwarten, dass künftig vor allem jüngere Politiker der Partei, die unter anderem mehr föderale Strukturen fordern, den Präsidenten, herausfordern werden. Der Haftbefehl des Internationalen Strafgerichtshofs gegen al-Bashir gilt langfristig als die größte Hypothek für den Sudan. Zudem stellt es für Khartoum eine schwerwiegende außenpolitische Belastung dar, dass der Sudan von den USA noch immer als Staat gelistet wird, der den internationalen Terrorismus unterstützt. ${ }^{32}$ Die USA haben zwar eine Streichung in Aussicht gestellt, falls die Trennung vom Südsudan friedlich vonstatten geht, bisher hat sich am Status des Sudan allerdings nichts geändert. Auch die damit verbundenen Sanktionen bleiben somit in Kraft.

In Darfur verlangen mehrere Rebellengruppen seit 2003 eine Beteiligung an der Macht. Seit Jahren finden immer wieder Verhandlungen statt, die eine nachhaltige Lösung zustande bringen sollen, diese sind bislang aber stets gescheitert. Dies liegt nur teilweise an den sehr gegensätzlichen Positionen der Verhandelnden. Viel wichtiger scheint die Heterogenität der verschiedenen Rebellengruppen, die nicht zuletzt lokalen Einflüssen unterliegen. ${ }^{33}$ Der Darfur-Konflikt hat zwar keinen direkten Einfluss auf die Beziehungen zwischen Sudan und Südsudan,

30 Siehe z.B. China to Loan South Sudan $\$ 8$ Billion for Infrastructure Projects, http://www.bloomberg.com/news/2012-04-28/china-to-loan-southsudan-8-billion-for-infrastructure-projects.html sowie South Sudan Hunts for Loans as Oil-Output Halt Dents Economy, http://www.businessweek. com/news/2012-05-10/south-sudan-negotiates-loans-as-oil-output-haltdents-economy,abgerufen am 07.01.2013.

31 How Sudan's Bashir survived the Arab Spring, http://www.aljazeera.com/ indepth/features/2012/09/2012921164748873959.html, abgerufen am 07.01.2013.

32 Siehe State Sponsors of Terrorism, http://www.state.gov/j/ct/list/c14151. htm, abgerufen am 07.01.2013.

33 Behrends, Andrea und Schlee, Günther. 2009: Lokale Konfliktstrukturen in Darfur und dem Osten des Tschad oder: Was ist ethnisch an ethnischen Konflikten. In: Feichtinger, Walter und Hainzl, Gerald (Hrsg.): Krisenmanagement in Afrika. Erwartungen - Möglichkeiten - renzen. Böhlau: Wien. dennoch könnte die daraus resultierende Instabilität das zwischenstaatliche Konfliktpotenzial verschärfen. Der Sudan hat seinem Nachbarstaat bereits vorgeworfen, Rebellen aus Darfur Rückzugs- und Nachschubmöglichkeiten zu gewähren. ${ }^{34}$

\section{Ausblick}

Eine Annäherung an und ein möglicher Beitritt des Südsudan zur East African Community (EAC) ist nicht nur durch die geplante Pipeline nach Lamu (Kenya) sehr wahrscheinlich, sondern auch die langfristigen wirtschaftlichen Beziehungen zu Kenya und Uganda, beide Gründungsmitglieder der EAC. Durch den Pipelinebau erhofft sich der Südsudan einerseits eine Reduktion der Abhängigkeit beim Ölexport vom Sudan und andererseits die Möglichkeit, zumindest zwischen zwei Anbietern auswählen und damit die Transitgebühren senken zu können.

Hinsichtlich der gemeinsamen Grenze und des Streits um Abyei wurden seit der Unabhängigkeit des Südsudan zwar einige Fortschritte erzielt, allerdings bahnt sich hier nicht wirklich eine Lösung an. Das für 2013 ins Auge gefasste Referendum könnte unter den derzeitigen Rahmenbedingungen am Widerstand des Sudan scheitern bzw. verschoben werden.

Die Auseinandersetzungen der beiden Staaten bergen nach wie die Gefahr einer gewaltsamen Eskalation, wie der Einmarsch des Sudan in Abyei oder der Angriff des Südsudan auf Heglig gezeigt haben. Eine Entwicklung zu einem zwischenstaatlichen Krieg ist aber, nicht zuletzt auch durch die starke internationale Präsenz, in absehbarer Zeit nicht wahrscheinlich. Dass beide Staaten Rebellengruppen der jeweils anderen Seite unterstützen, hat eine gewisse Tradition und könnte auch in Zukunft als Mittel zur Destabilisierung eingesetzt werden.

Abseits von kurzfristigen Rückschritten hat sich die Situation über einen längeren Zeitraum jedoch durchaus positiv entwickelt. Wer hätte vor zehn Jahre eine relativ gewaltlose Trennung der beiden Landesteile vorausgesehen? Zudem bleiben die beiden Staaten trotz ihrer Trennung in ökonomischer und sozialer Hinsicht in vielfacher Weise miteinander verknüpft. Die durch die Unabhängigkeit des Südsudan entstandenen Probleme müssen entweder rational gelöst oder, wenn dies aufgrund der historischen Belastungen nicht möglich ist, über einen längeren Zeitraum hinweg durch Mediation bzw. Krisen- und Konfliktmanagement begleitet werden.

34 Diesbezügliche Äußerungen von Vertretern des Sudan müssen jedoch mit Vorsicht bewertet werden. Dass das Staatsgebiet des Südsudan genutzt wird, muss nicht notwendigerweise auf eine Unterstützung hindeuten, sondern kann auch Ausdruck dafür sein, dass der Südsudan nicht in der Lage ist, sein Staatsgebiet zu kontrollieren. 